\title{
Metabolic syndrome and non-alcoholic fatty liver disease in post-transplant patients affected by hepatitis $\mathrm{C}$ virus (genotype 1)
}

\author{
Gianni Testino $\cdot$ Paolo Borro
}

Received: 28 June 2010/ Accepted: 7 July 2010/Published online: 28 July 2010

(C) Springer-Verlag 2010

\begin{abstract}
Metabolic syndrome and allograft non-alcoholic fatty liver disease (NAFLD) is a common occurrence after orthotopic liver transplantation (OLT). NAFLD and hepatitis $\mathrm{C}$ virus (HCV) are frequently associated. The association steatosis/HCV determines important implications for clinical practice: steatosis accelerates the progression of fibrosis and reduces the likelihood of obtaining a sustained virological response (SVR) with antiviral therapy. We have evidenced a strong correlation between body mass index (BMI), cholesterol, triglycerides (TGC) and hepatic percentage of steatosis. In subjects with $\mathrm{BMI}<25$ and TGC $<160 \mathrm{ng} / \mathrm{ml}$, the chance of SVR was 48 times higher than that of non-response. The chances of SVR for patients with percentage of steatosis $<15$ were 12 times higher than that with higher percentage of steatosis value. Detection, prevention and management of metabolic disorders and NAFLD should be one of the primary objectives of all transplant teams.
\end{abstract}

Keywords Hepatitis C virus - Metabolic syndrome .

Non-alcoholic fatty liver disease .

Orthotopic liver transplantation

Changes in daily life, free food intake, normalization of the hypermetabolic state of end-stage liver disease and the metabolic adverse effects of immunosuppression may lead to development of metabolic disturbances characterizing the metabolic syndrome (3 or more of the ATP III criteria) after orthotopic liver transplantation (OLT) [1]. In the

G. Testino $\cdot$ P. Borro $(\bowtie)$

Department of Specialistic Medicine, S. Martino Hospital, Genoa, Italy

e-mail: pborro@yahoo.com experience of Bianchi et al. [2], the prevalence of the metabolic syndrome was assessed in 296 liver transplant patients in the course of regular follow-up at least 6 months after OLT. Overall the metabolic syndrome was present in $44.5 \%$ of patients and insulin resistance was observed in $41 \%$ of cases.

Immunosuppressive medications play a role in the pathogenesis of post-transplant diabetes mellitus and other metabolic disturbances. Calcineurin inhibitors impair insulin sensitivity and reduce insulin secretion. Thus, both cyclosporine and tacrolimus have diabetogenic activity. Diabetes is more commonly associated with tacrolimus, whereas hypertension and hyperlipidemia tend to be more prominent with cyclosporines [1].

The most important clinical consequences of the metabolic syndrome are the risk of developing cardiovascular diseases and non-alcoholic fatty liver disease (NAFLD) [1].

NAFLD due to metabolic syndrome affects nearly $40-50 \%$ of all transplant recipients and cannot be ignored $[1,3,4]$. NAFLD is characterized by the accumulation of fat droplets in hepatocytes. In early NAFLD, the fat is seen in zone 3 hepatocytes. The spectrum of NAFLD extends from hepatic steatosis through steatohepatitis to cirrhosis.

NAFLD and hepatitis $\mathrm{C}$ virus (HCV) are frequently associated diseases. Epidemiological and experimental data indicate that $\mathrm{HCV}$ predisposes to NAFLD and the prevalence of coexistent hepatitis $\mathrm{C}$ and steatosis is two to three times higher than it would be expected if these were completely independent processes.

Watanabe et al. [5] evidenced that several metabolic disturbances, such as obesity, insulin resistance and hepatic steatosis, are significant risk factors for decreased sustained virological response (SVR) to the interferon and ribavirin combination antiviral therapy in chronic hepatitis $\mathrm{C}$ 
patients. It should be evidenced, for completeness, the influence of non-alcoholic fatty liver disease (NAFDL) on post liver transplantation (LT) antiviral treatment $[2,4]$.

It is well known how the natural history of recurrent hepatitis $\mathrm{C}$ is accelerated after LT. Hepatitis C driven fibrosis response in the allograft leads to the development of graft cirrhosis in approximately $25 \%$ of recipients after a followup of 5-10 years. More than $10 \%$ of patients who undergo transplantation for hepatitis $\mathrm{C}$ cirrhosis eventually require retransplantation for hepatitis C-related graft failure [6].

In relation with organ shortage, Llado et al. [7] affirmed that retransplantation is not an option for recurrent hepatitis C cirrhosis after LT. For this reason, it is mandatory to protect in the best way the graft by treating with antiviral therapy the recurrent hepatitis $C$ [8].

In a substantial proportion of infected recipients, liver injury remains mild despite high viral burden. Several factors have been clearly shown to be associated with fibrosis progression rate. Metabolic conditions and steatosis are emerging as independent cofactors of fibrogenesis [9].

Till date in our experience only the importance of parameters such as body mass index (BMI, $\mathrm{kg} / \mathrm{m}^{2}$ ), cholesterol (mg/dl), triglycerides (TGC, $\mathrm{ng} / \mathrm{ml})$ and hepatic percentage of steatosis in the response to therapy with pegylated interferon (PEG-IFN) alfa-2b and ribavirin in patients with recurrent hepatitis $\mathrm{C}$ after LT has been evidenced [10].

Response to therapy was evaluated in relation to virological response (at 1-3-6-12 and 18 months HCV-RNA assay was performed): SVR was defined as the absence of serum HCV-RNA (virological remission) and serum alanine aminotransferase (ALT) activity within the reference range for at least 6 months; sustained biochemical response (SBR) was defined as a decrease in serum ALT activity within the reference range but with reappearance or persistently detectable serum HCV-RNA; non-response (NR) was defined as persistence/relapse at the end of therapy of HCV-RNA and no decrease in ALT activity or relapse at the end of therapy.

10 patients $(31.2 \%)$ stopped therapy due to side effects; SVR was observed in 6 cases $(6 / 22,27.2 \%)$, SBR in 7 cases $(7 / 22,31.8 \%)$ and NR in 9 cases $(9 / 22,40.9 \%)$.

The observed distributions of BMI, cholesterol, TGC and percentage of steatosis were confirmed to be normally distributed by the one-sample Kolmogorov-Smirnov Goodness of Fit test procedure. Comparison of BMI, cholesterol, TGC and percentage of steatosis between NR, SVR and SBR groups were analyzed by ANOVA with a post hoc Bonferroni test and correlation between variables was tested by Pearson test. The univariate analysis was performed to estimate the chance of response on the basis of the above mentioned variables.
BMI, TGC, percentage of steatosis in NR group $(26.8 \pm 3.3,245.3 \pm 84.4$ and $26.8 \pm 23.6$, respectively $)$ were higher than the values observed in SVR $(20.4 \pm 2.6$, $p$ value $<0.001 ; \quad 108.3 \pm 48.4, p \quad$ value $=0.002$ and $5.75 \pm 2.2, \quad p$ value $=0.027$, respectively) and SBR groups $\quad(21.5 \pm 1.6, \quad p \quad$ value $=0.003, \quad 132.4 \pm 51.2$, $p$ value $=0.008,6.2 \pm 2.4, p$ value $=0.033$, respectively). The differences between SVR and SBR were not significant for all the above mentioned variables. No significant differences were registered in NR (197.6 \pm 47.3$)$, SVR $(149.3 \pm 37)$ and SBR $(153.4 \pm 41.5)$ groups for cholesterol.

Pearson correlation test (Correlation coefficient $>0.7$, $p<0.001$ for every correlations) showed a strong correlation between BMI, cholesterol, TGC and percentage of steatosis.

For patients with BMI $<25$ and TGC $<160$, the chance of SVR was 48 times higher than that of NR. The chances of SVR and SBR responses for patients with percentage of steatosis $<15$ were 12 times higher than that with higher percentage of steatosis values.

The observed diminished response has been hypothesized to be due to decreased interferon bioavailability in overweight patients, presence of hepatic steatosis, which itself is a predictor of poor response to antiviral treatment [8].

Moreover, increased expression of suppressor of cytokine that inhibit interferon signaling may be one mechanism by which overweight reduces the interferon response [11].

Some authors evidenced how the possible altered immune function in obesity may be mediated by leptin resistance. Leptin is an important modulator of the Th-1 response [12]. It is possible that patients with steatosis resulting from insulin resistance, and not virally mediated, may be unable to up-regulate adequately the Th-1 response via IFN because of underlying significant leptin resistance. This could lead to altered viral clearance and a decrease in SVR [12].

It is well known how the retransplantation is not an option for recurrent hepatitis C cirrhosis after LT; therefore, from our experience there emerges the importance of a better histological and internistic evaluation in order to reach the highest number of SVR in patients with recurrent hepatitis $\mathrm{C}$.

In post-transplant subjects affected by $\mathrm{HCV}$, the clinical impact of the metabolic disturbances and NAFLD is one of the most important clinical entity that should be taken into consideration.

Kadayifci [1] affirms that all post-transplant patients should be advised about how to maintain a calorie and nutrient-balanced diet and an optimal exercise program; metabolic disturbances (hypertension, dysplipidemia and 
diabetes) should be treated aggressively; life style modification (loss of weight, increase in physical activity and healthy diet) should be obeyed strictly $[1,13,14]$.

We can conclude how the amount of steatosis be noted specifically in biopsy examination reports of patients with relapse chronic hepatitis $\mathrm{C}$ and how the management of dismetabolism, diet and exercise therapy can improve BMI, liver histology and, therefore, the response to antiviral therapy $[15,16]$.

Detection, prevention and management of metabolic syndrome and NAFLD should be one of the primary objectives of all transplant teams.

Conflict of interest None.

\section{References}

1. Kadayifci A (2009) Metabolic syndrome and liver transplantation. Panminerva Med 51:205-213

2. Bianchi G, Marchesini G, Marzocchi R et al (2008) Metabolic syndrome in liver transplantation: relation to etiology and immunosuppression. Liver Transpl 14:1648-1654

3. Testino G, Sumberaz A (2007) Liver steatosis and antiviral therapy in chronic hepatitis C. Liver Int 27:287

4. Shifflet A, Wu GY (2009) Non-alcoholic steatohepatitis: an overview. J Formos Med Assoc 108:4-12

5. Watanabe S, Yaginuma R, Ikejima K, Miyazaki A (2008) Liver steatosis and metabolic syndrome. J Gastroenterol 43:509-518

6. Gane E (2002) Treatment of recurrent hepatitis C. Liver Transpl 8(Suppl 1):S28-S37
7. Llado L, Castellote J, Figueras J (2005) Is retransplantation an option for recurrent hepatitis $\mathrm{C}$ cirrhosis after liver transplantation? J Hepatol 42:468-472

8. Perumalswami P, Kleiner DE, Lutchman G et al (2006) Steatosis and progression of fibrosis in untreated patients with chronic hepatitis C infection. Hepatology 43:780-787

9. Machicao VI, Krishna M, Bonatti H et al (2004) Hepatitis C recurrence is not associated with allograft steatosis within the first year after liver transplantation. Liver Transpl 10:599-606

10. Testino G, Sumberaz A, Ravetti GL et al (2006) Pegylated interferon (PEG-IFN) alfa-2b and ribavirin for recurrent hepatitis C (genotype 1) after liver transplantation: relationship with steatosis. Dig Liver Dis 38:860-861

11. Walsh MJ, Jonsson JR, Richardson MM et al (2006) Non response to antiviral therapy is associated with obesity and increased hepatic expression of suppressor of cytokine signalling 3 in patients with chronic hepatitis $\mathrm{C}$, viral genotype 1. Gut 55:529-535

12. Bahr MJ, Manns MP (2003) Recurrent hepatitis C in transplanted patients: more questions than answers. Dig Liver Dis 35:2-6

13. Speliotes EK, Massaro JM, Hoffmann U et al (2010) Fatty liver is associated with dyslipidemia and dysglycemia independent of visceral fat: the Framingham hearth study. Hepatology 51:1979-1987

14. Lewis JR, Mohanty SR (2010) Nonalcoholic fatty liver disease: a review and update. Dig Dis Sci 55:560-578

15. Testino G, Sumberaz A, Ancarani O et al (2009) Influence of body mass index, cholesterol, triglycerides and steatosis on pegylated interferon alfa-2a and ribavirin treatment for recurrent hepatitis $\mathrm{C}$ in patients transplanted for $\mathrm{HCV}$ and alcoholic cirrhosis. Hepatogastroenterology 56:501-503

16. Testino G (2005) Steatosis and hepatitis C. Recenti Prog Med 96:621-633 